\title{
Towards Completeness and Lawfulness of Business Process Models
}

\author{
Ludmila Penicina and Marite Kirikova \\ Institute of Applied Computer Systems, Riga Technical University, 1 Kalku, Riga, \\ LV-1658, Latvia \\ \{ludmila.penicina, marite.kirikova\}artu. $1 \mathrm{v}$
}

\begin{abstract}
We address the existing gaps between business process models, enterprise architecture (EA) models and, external regulations that hinder completeness and lawfulness of business process models. As a solution we propose a high-level architecture for business process knowledge management that bridges the identified gaps. We use de-facto industry standards for modelling business processes and EA - BPMN and ArchiMate. We propose to use BungeWand-Weber (BWW) model as a theoretical foundation for storing business process knowledge represented in BPMN and ArchiMate models and storing elements that address lawfulness of the models. BWW model provides a framework for systematically storing internally maintained process knowledge (models) and externally maintained knowledge (regulations), and supporting completeness and lawfulness of the models. Thus, the main contributions of our approach is supporting completeness and lawfulness of business process models and supporting the creation of information services to increase efficiency in the business process modelling context.
\end{abstract}

Keywords: Business process modelling, BPMN, ArchiMate, BWW.

\section{Introduction}

Business processes are valuable assets of any organization as they contain vital organizational know-how knowledge. According to [1] knowledge is the most strategically important resource of the firm and primary role of any organization is application of knowledge in its everyday activities. However, the application of existing business process knowledge has always been a sophisticated task. Business process knowledge must be reusable and applicable across many business processes [2]. The need for business process knowledge reuse is driven (1) by the challenge to build accurate, complete and compliant with regulations business process models, (2) by the need to facilitate common understanding of processes, and (3) by intention to save the time and resources for business process modelling and analysis. Business process knowledge can be stored in internal artefacts of enterprise (e.g., business process models and enterprise architecture models) and externally maintained documents (e.g., legislation, regulations). One of the most important issues in business process modelling is to create models that are consistent with legislative and regulatory 
documents (for simplicity further in the text we call these documents "regulations") and to provide valuable information services for monitoring of changes in regulations.

Nowadays organizations employ industry modelling standards like BPMN [3] to understand and improve business processes. However, BPMN models are only one component of business modelling required for a holistic view of end-to-end business processes. More information is needed to build information systems supporting organizational business processes [2]. To create complete business process models additionally to the process flow aspect it is necessary to consider the following aspects of organizations:

- Structural aspects such as actors, data objects, existing application landscape, network infrastructure, etc. Therefore Enterprise Architecture (EA) models that can reflect these aspects are an essential component of creating accurate and complete business process models and must be linked with business process models.

- Vital process knowledge (like lawful states and events) reflected in externally maintained regulations. Therefore real-time linkage with regulations is essential to support lawfulness of the models and monitoring of changes.

The aforementioned requirements were acquired empirically during the joint research project (contract No. 5/7-2012) with Ltd "Komerccentrs DATI grupa", Latvia. During the project the case study creating an enterprise model process patterns for the Latvian Accounting Law was carried out (published in [4]). The obtained results showed the limitations of BPMN concerning structural modeling and modeling of lawful aspects of the system.

Thus, building complete and accurate business process models requires maintaining the relationships with EA models and externally maintained regulations related to business process models. A growing number of different initiatives and projects (like [5] and [6]) that support implementation of a machine-readable set of technologyneutral electronic representations (in XML format) of regulations show that regulations maintenance moves forward to making open and "visible" the structure and semantic components of regulations so as to support the creation of high value information services to increase efficiency in different every-day contexts. Making regulation structure "visible" and machine-readable opens a potential for linking external regulations with internal artefacts like business process models and, namely, with BPMN 2.0 process models. The new BPMN 2.0 specification [3] allows integration with third party components using XML-based representation languages (e.g., OWL, RDF) [2]. We envision that initiatives to make regulations machine-readable and "visible" will grow world-wide in coming years.

BPMN standard itself does not allow to link business process model with other models of enterprise architecture, however, many so-called Business Process Analysis (BPA) suites allow to do it [2]. ArchiMate enterprise architecture modelling language has been developed to provide a uniform representation for diagrams that describe enterprise architectures [7]. Here the existence of business processes model is depicted. However, ArchiMate does not prescribe to list the flow of activities in detail. Linkage between business process models and EA models would allow to look at the business processes at different layers of the enterprise in detail and to describe artefacts and actors related to the business process at different enterprise layers. However, 
unlike BPMN 2.0 standard ArchiMate 2.0 specification does not describe elements that support integration with externally defined third party artefacts.

This paper presents an on-going research which aims to propose architecture for business process knowledge management providing linkage of business process models, EA models and externally maintained regulations for efficient business process modelling supporting completeness and lawfulness of the models. To provide an efficient environment for business process modelling that allows (1) linking process models with EA models and external regulations and (2) systematically storing and retrieving business process knowledge represented in BPMN and ArchiMate models we propose to use a meta-structure as the foundation for business process knowledge storage. We propose to use Bunge-Wand-Weber (BWW) model [8] as the metastructure for business process knowledge and its management. BWW model consists of constructs that are present in the real world and must be represented in information system [9], including lawful and unlawful states and events that are an important issue in regulations.

The potential practical implications of the proposed approach can be summarized as follows:

- It allows systematically storing and reusing the knowledge contained in BPMN, ArchiMate models and externally defined regulations.

- It provide the frame for complete and regulations complaint representations of the system being modeled.

- It allows describing lawful and unlawful aspects of information systems not addressed in BPMN and ArchiMate models.

- It allows describing conceivable event and state space of information systems reducing the possibility to include non-existing ("unconceivable") states in the models.

The paper is structured as follows. In Section 2 the related work is outlined. In Section 3 existing gaps in providing completeness and lawfulness of business process models are discussed. Section 4 describes the proposed solution. In section 5 an example of the proposed solution is discussed. Brief conclusions and future work are presented in Section 6.

\section{$2 \quad$ Related Works}

BWW model [7] extends the systems ontology presented by Mario Bunge [10]. Wand and Weber developed it as a formal foundation for modelling information systems consisting of the constructs present in the real world that must be represented in information system [8]. BWW model is an upper level ontology containing general concepts that are necessary for description of information systems [9]. Elements in BWW model can be grouped in the following groups (adapted from [9]; the elements of BWW model are shown in italics):

1. Things - including Properties, Classes and Kinds of Things. Thing is an elementary unit in BWW. Things possess Properties, which defines States of a Thing. Things can belong to Classes or Kinds depending on a number of common Properties. A Thing can act on another Thing if its existence affects the History of the other Thing. Things are coupled if one Things acts on another. 
2. States of Things - Properties of Things define their States. State Law restricts Values of Properties of Things. Conceivable State Space is a set of all States a Thing can assume. Lawful State Space defines States that comply with State Law. Stable State is a State in which a Thing or a System will remain unless forced to change by a Thing in the System Environment. Unstable state is a State that will be changed into another State by the Transformations in the System. History is the chronologically-ordered States of a Thing.

3. Transformations - transformation between States of Things. Transformation is a mapping from one State to another. Lawful Transformation defines which Events in a Thing are lawful.

4. Events - event is a change in State of a Thing. Conceivable Event Space is a set of all Events that can occur to a Thing. Lawful Event Space is a set of all Events that are lawful to a Thing. Events can be Internal Events and External Events. Events can be Well-Defined - an Event in which the subsequent State can be predicted or Poorly-Defined - an Event in which the subsequent State cannot be predicted.

5. Systems - a set of coupled Things. System Composition are Things in the System. System Environment are Things outside the System interacting with the System. System Structure is a set of couplings that exists among Things. Subsystem is a System whose composition and structure is a subset of the composition and structure of another System. System Decomposition is a set of Subsystems. Level Structure is an alignment of the subsystems.

The BWW model has been used in a number of studies for evaluation of modelling techniques. The authors of [9] report on the outcomes of an ontological analysis of BPMN and explore identified issues by reporting on interviews conducted with BPMN users in Australia. As a result [9] defines few potential shortcomings in BPMN - such as existence of some ambiguous elements in its specification. The authors of [11] propose a comprehensive Conceptual Modelling Quality Framework based on BWW model bringing together and organizing the various quality cornerstones and then defining many quality dimensions that connect one to another. The authors of [12] examine how process modelling techniques have developed and compare modelling techniques using BWW model as a benchmark used for the analysis of grammars that purport to model, the real world, and the interactions within it. The authors of [13] propose an approach for developing a conceptual model that represents the structural, relational and behavioural elements of the computing systems based on the BWW model. The authors of [14] use of the BWW model to compare the representation capabilities of two business rule modelling languages. We selected the BWW model for this study for the following reasons (a) BWW model was created for Information Systems discipline, (b) because of BWW foundational quality BWW model is an upper ontology describing generic elements that must be present in every information system, (c) there is a significant research track record of using BWW.

Authors of [15] present a framework for business process repositories that assists in managing large collections of process models. The authors emphasize that BPMN models repository should be stored in XML format and ontologies must be stored in repository that will facilitate integration of processes from multiple parties. 
A number of initiatives and researches exist for presenting legislative and judiciary documents in machine readable format to support automatic retrieval interoperability. AKOMA NTOSO schema [6] is a set of XML machine-readable descriptions of official documents such as legislation, debate record, minutes, etc. that allow exchanging and reusing parliamentary, legislative, and judiciary documents more efficiently enabling addition of descriptive structure (markup) to the content of parliamentary and legislative documents [6].

Authors of [16] provide a high-level architecture of the document analysis and change detection system which is used for the retrieval of regulations and document analysis and preparation for their linkage to business processes. Authors of [4] report on enterprise modeling experiment that is based on representation of regulations as reusable business process model parts. Their experiment showed that for proper positioning of the parts it is necessary to represent in models not only the process per se, but also other related information available in regulations [4]. Authors of [17] envision an approach where regulations are translated into business process model "spare parts" or raw materials that can be used by designers of business processes at several enterprises (or several units in one enterprise).

Related works have encouraged this research and showed that BWW model can be used for the analysis of the completeness of knowledge about the enterprise and its information systems, in general, as well as of business processes, in particular.

\section{Existing Gaps in Providing Completeness and Lawfulness of Business Process Models}

This section presents shortcomings of existing standards and methods in relation to abilities to present a complete business process description compliant with regulations and store business process knowledge contained in business process models.

Figure 1 depicts existing gaps in supporting completeness and lawfulness of business process models in different domains of business process knowledge, namely, the lack of standardized linkage between BPMN models and ArchiMate models and the lack of linkage between BPMN, ArchiMate, and regulations to provide lawfulness of the models. These gaps hinder completeness and lawfulness of business process and EA models - without such linkage lawful and unlawful aspects of the system are not depicted in the models and thus models may contain meaningless states and events, since a set of conceivable states and events also are not depicted. Without a meaningful coupling between EA, business process models, and regulations, the models are isolated, potentially not compliant with regulations, inconsistent, and conflicting.

Some tools like ARIS [18] allow augmenting BPMN and ArchiMate models in their modelling environments, however, these are just tool vendor initiatives and there exist no standardized linkage between BPMN and ArchiMate. Linkage between BPMN and regulations is still a challenge. However the new BPMN 2.0 specification [3] allows integration with third party components using XML-based representation languages (e.g., OWL, RDF) [2]. This new BPMN 2.0 "plug-and-play" feature opens the potential for linking external resources represented as XML structures with 
BPMN 2.0 models. But, to utilize it, it is necessary to provide a foundation for such linking - a consistent meta-structure for storing and reusing business process knowledge contained in business process models, enterprise architecture models, and external documents such as XML-based regulations. In ArchiMate 2.0 specification there is no description of how ArchiMate 2.0 models can be linked with external resources.

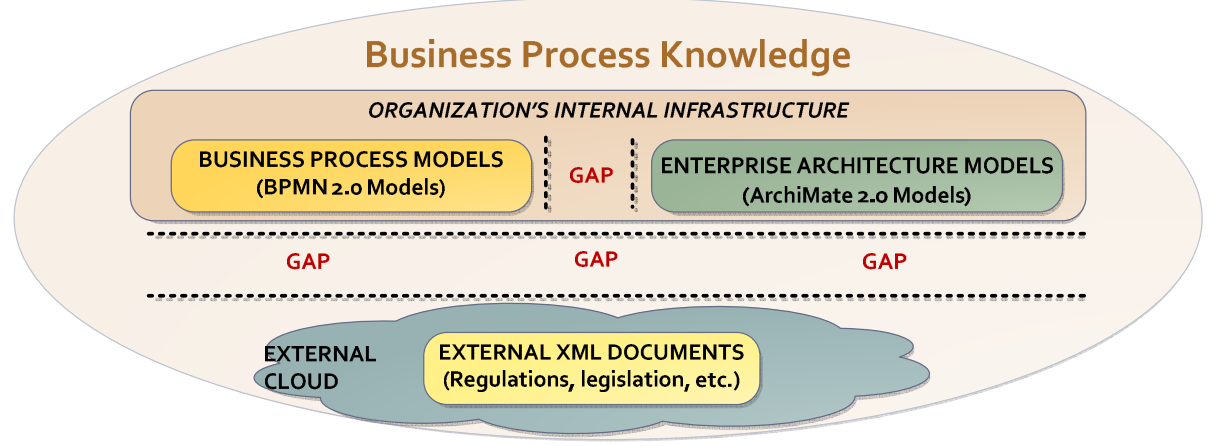

Fig. 1. Existing gaps between BPMN, ArchiMate and externally maintained regulations

To best of our knowledge there is no research papers available that close the gap in linking BPMN, ArchiMate and externally maintained XML-based regulations and managing business process knowledge based on BWW model. Since BWW model describes the elements that must be addressed in every system, including lawful and unlawful states and events of the system; we propose to use BWW as a meta-structure for storing business process knowledge depicted in EA models, BPMN models, and regulations.

\subsection{BPMN Limitations}

Business Process Model and Notation (BPMN) [3] is the de-facto standard for representing in a very expressive graphical way the processes occurring in virtually every kind of organizations [19]. However BPMN has its limitations when it comes to modelling other aspects of organizations such as organizational structure and roles, functional breakdowns, data, strategy, business rules, and technical systems [2]. Thus BPMN alone cannot provide complete business process descriptions.

Research presented in [9] describes which BPMN elements correspond to BWW model. There are $6 \mathrm{BWW}$ model elements that are not supported by BPMN notation, namely, State law, Conceivable State Space, Lawful State Space, History, Conceivable Event Space, and Lawful Event Space. Since BWW model describes aspects that are important for building information systems [9], these six elements are to be taken into consideration to define a complete, lawful and consistent description of business processes. 


\subsection{ArchiMate Limitations}

Architecture descriptions are formal descriptions organized in a way that supports reasoning about the structural and behavioural properties of the system and its evolution [7]. To provide a uniform representation for diagrams that describe enterprise architectures, the ArchiMate enterprise architecture modelling language has been developed [7]. It offers an integrated architectural approach that describes and visualizes the different architecture domains and their underlying relations and dependencies [7]. ArchiMate 2.0 is a modelling language that provides graphical representations of enterprise architecture based on TOGAF standard [20].

ArchiMate 2.0 language defines 3 layers of an enterprise architecture [7]:

1. Business layer offers products and services to external customers.

2. Application layer supports the business layer with application services.

3. Technology layer offers infrastructure services needed to run applications.

In an ArchiMate model, the existence of business processes is depicted. However, it does not prompt to represent the flow of activities in detail. During business process modelling, a business process can be expanded using a business process design language; e.g., BPMN [3].

ArchiMate modelling language does not support 11 concepts of BWW model, namely, State, Conceivable state space, Lawful state space, State law, Stable state, Unstable state, History, Conceivable event space, Lawful event space, Well-defined event, Lawful transformation. Unlike in BPMN, ArchiMate does not define specific event types in which subsequent State can always be predicted. Although the ArchiMate modelling language does not support the description of 11 BWW elements, which is more than $6 \mathrm{BWW}$ elements missing in BPMN, the ArchiMate allows defining structural components of an information system at all three levels of the enterprise architecture in much greater detail than BPMN.

\subsection{Business Process Repositories Limitations}

Most business process management platforms provide business process repositories for managing knowledge contained in business process models.

Based on literature analysis we have identified the following shortcomings of existing business process repositories:

- Most of existing repositories for storing business process models and related knowledge are based on Relational DBMS, however in case of storing knowledge contained within BPMN models it is preferable to use XML based structures for storing, e.g., XQuery, since BPMN formal meta-model is defined using XML [3].

- The authors of [15] state that BPMN Repository based on XML database and ontologies is rather an architecture than a practical implementation.

Existing methods for storing knowledge of business process models do not address the issue of linking business process models with EA models, linking business process models with externally maintained XML based regulations, and structuring the 
knowledge flexibly according to various systems of the enterprise. We propose to use BWW model as the foundation for business process repository and build business process repository using XML based structures to support process models linkage with external structures.

\section{Proposed Solution}

This section describes proposed solution to bridge existing gaps in business process knowledge management by using BWW model as a meta-structure for storing business process knowledge. We employ BWW model as a foundation for linking BPMN, ArchiMate and externally (to business process repository) maintained XML-based regulations.

Figure 2 depicts a high-level architecture for the proposed solution. Our approach provides the following:

- Linking externally maintained XML structured regulations with BPMN 2.0 models - using the new BPMN 2.0 "plug-and-play" functionality to integrate with the third party XML based documents.

- Proposing an extension for ArchiMate 2.0 standard to enable linking ArchiMate 2.0 models with the third party XML based documents.

- Using existing research (e.g., [21]) to enhance BPMN 2.0 and ArchiMate 2.0 integration to provide a valuable mapping and linkage between these two complementary standards.

- Storing in BWW based XML structured repository business process knowledge from BPMN and ArchiMate models with descriptions of lawful and unlawful aspects of the system depicted from regulations as a result of the linkage.

ArchiMate models complement BPMN models with different layers of enterprise architecture. External regulations complement BPMN and ArchiMate models with lawful and unlawful states and events of the system. A meta-structure (background knowledge) is necessary to maintain the relationships between BPMN models, EA models, and external regulations. If BWW model is used as a meta-structure for storing knowledge about the business process, and if BPMN and ArchiMate are used as languages of representation of this knowledge; it is necessary to identify which elements from BWW model are supported by BPMN and ArchiMate models. BWW model defines elements of the information system that are supported by BPMN and ArchiMate standards as well as a set of elements that are not supported by these standards but are depicted in regulations and must be addressed in models to support lawfulness of the system (e.g., lawful states, lawful events). Majority of BPMN and ArchiMate core elements can be mapped to BWW constructs. However BPMN models do not cover 6 BWW elements and ArchiMate models do not cover $11 \mathrm{BWW}$ elements. BPMN provides elements that support BWW model elements that are not supported by ArchiMate modelling language. Linked BPMN models with ArchiMate models cover more BWW elements than ArchiMate models can cover separately. 
Still there exist six elements that cannot be represented using interlinked BPMN and ArchiMate modelling languages, namely, State Law (SL), Conceivable State Space (CSS), Lawful State Space (LSS), History (H), Conceivable Event Space (CES), and Lawful Event Space (LES). It is necessary to address these missing elements in order to support the lawfulness of the system and eliminate non-existing ("unconceivable") states from the models. Using BWW model based business process knowledge repository will allow to describe these elements and to support creating ArchiMate and BPMN models compliant with regulations.

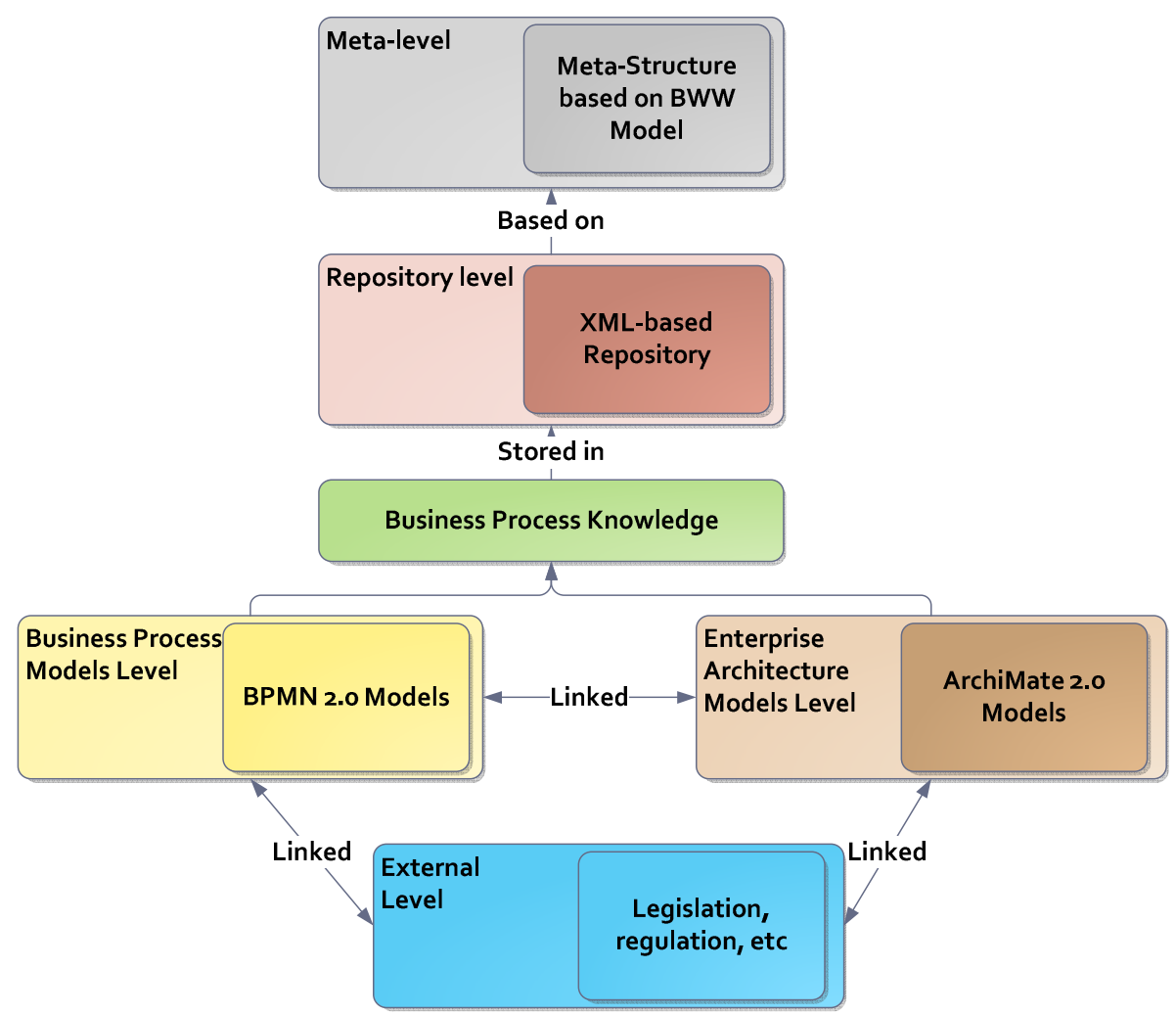

Fig. 2. The proposed high-level architecture for closing existing gaps between BPMN, ArchiMate and externally maintained regulations

\section{Example}

Figure 3 shows a BPMN model of publication self-archiving process (or Green Open Access) at University. This process provides a possibility for researchers to publish their works as full texts in Open Access online repository. Two of the most important steps of this process are: 
- Researchers must choose a licence under which they wish to publish their publication (activity "Choose CC Licence"). For this process we use a set of ready licences provided by Creative Commons (CC) organization [22]. CC licences are available online and in a machine-readable format which unlocks possibilities to use these licences in web-services.

- Researchers must choose a version of the full text which the publisher permits to archive in the institutional repository. The possible versions of the publication's full texts are: Pre-print, Post-print or Published version.

Figure 4 shows ArchiMate 2.0 model for a self-archiving process at University. This model contains 3 layers of enterprise architecture, namely, (1) business layer with actors, assigned roles to the actors and a high-level business process description, (2) application level with application components and application services used by the business level, and (3) technological level with API service and systems software used by the application layer. ArchiMate model complements BPMN business process model with actors: we can conclude that Librarian has the role of Systems Administrator that manages the publications and also we can see application and technological services used in the process. Actors and services are not present in BPMN model. ArchiMate 2.0 model contains only high-level definition of the process while BPMN model describes the process in details. Linking BPMN and ArchiMate models will contribute to better understanding of the process and its components.

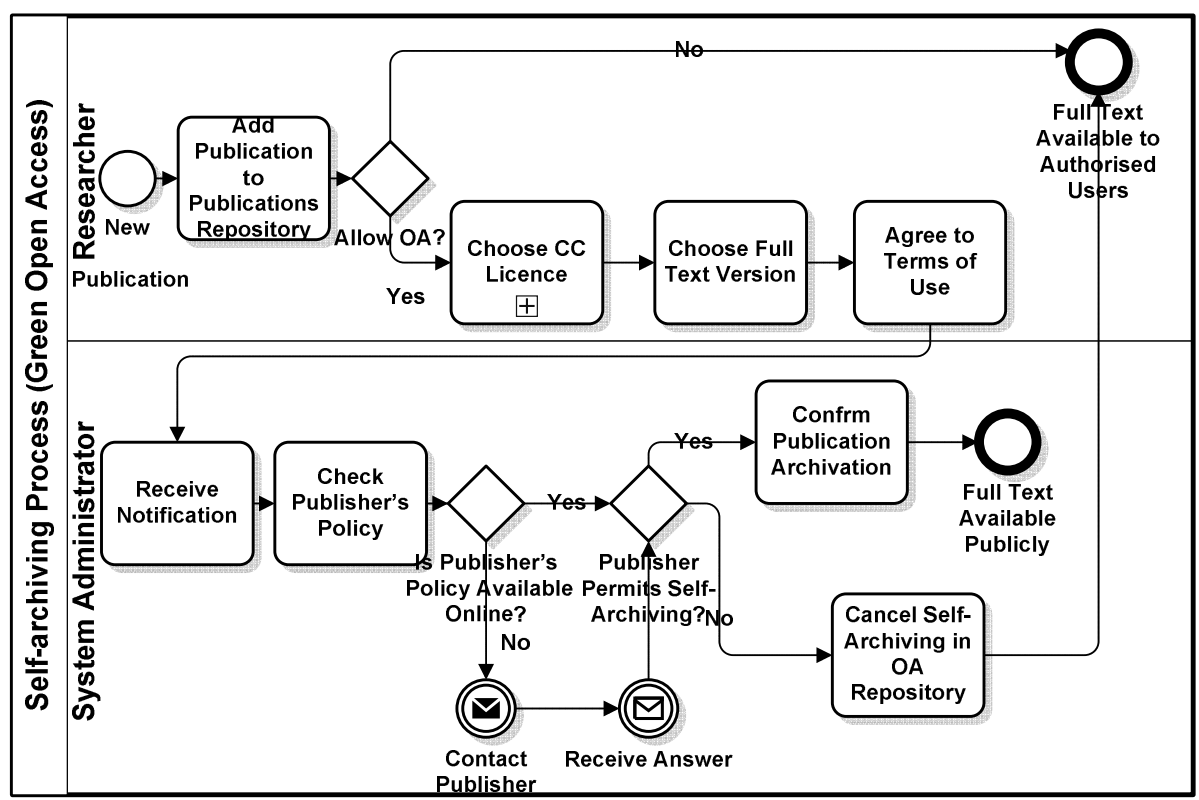

Fig. 3. BPMN 2.0 model of the self-archiving business process 


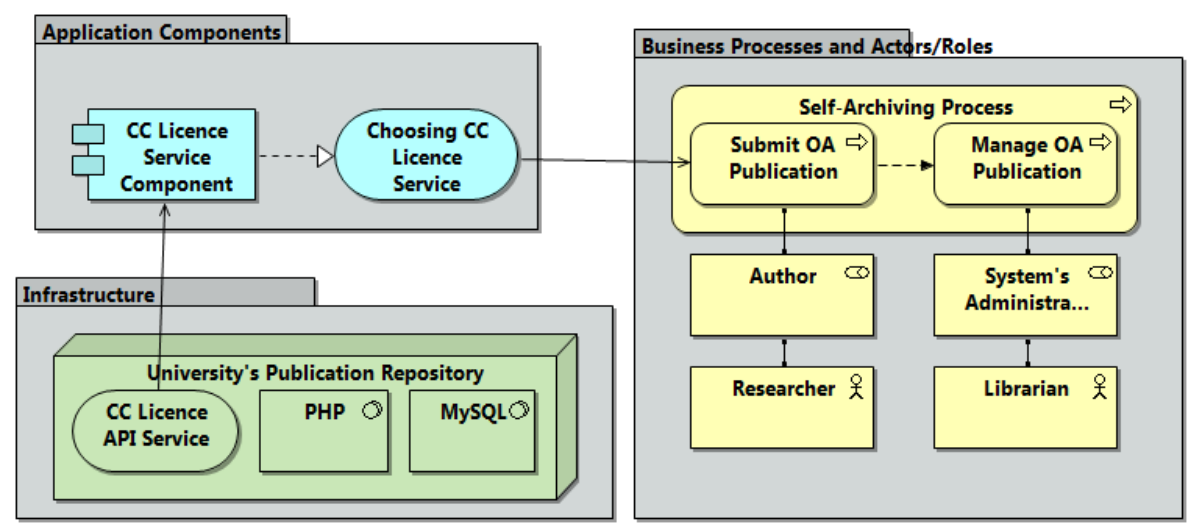

Fig. 4. ArchiMate 2.0 model of the self-archiving process

ArchiMate model supplements BPMN model with business, application and technology layers of the enterprise, still these models do not include descriptions of:

1. State Law - a set of all properties that are lawful to a Publication. We assume that a Publication can have the following properties: Version of the Full Text, MetaData, Licence, and Status. Each of the mentioned properties has a set of the values that are lawful, e.g., in this process it is important to understand what version of the full text the Author has submitted so the System Administrator can check if it corresponds to the Publisher's policy. Values for the Property "Version of the Full Text" would be \{Pre-print, Post-print, Published version\}, values for the Property "Status" would be \{Full Text Available to Authorised Users, Full Text Available Publicly\}, etc. However neither BPMN nor ArchiMate allows to define sets of lawful Publication's properties.

2. Conceivable State Space for a Publication can correspond to the situation where Publication's full text would be available publicly in case when Publisher's policy does not permit it. For example, Author has submitted post-print version of the full text but Publisher permits publishing only pre-prints - this state is Conceivable but it is Unlawful.

3. Lawful State Space for a Publication would correspond to the situation when the availability of the version of the full text of Publication publicly conforms to Publisher's policy.

4. History of states for a Publication would include all states of the publication in the process, e.g., including the past state Full Text Available to Authorised Users and the present state Full Text Available Publicly.

5. Conceivable Event Space can correspond to the situation when Systems Administrator does not receive a notification about a new Publication or the notification is sent out to the wrong employee, another Conceivable but Unlawful event might be when a Publisher does not reply to the System's Administrator request to permit publishing.

6. Lawful Event Space can correspond to the following events: Author submits a Publication, System's Administrator receives a notification about a new Publication, and the Publisher replies to the System's Administrator request. 
If the regulations to which the models must comply are available as external XML structures, it is possible to link BPMN process model elements with them. Figure 5 shows a part of an expanded sub-process for the activity "Choose CC Licence". In this sub-process the researcher chooses the appropriate CC licence. Linking each type of the licence with their online XML description will unlock possibilities to develop different information services for business process modeller, e.g., monitoring changes introduced to these licences and notifying responsible employees. This will contribute to promptly reacting to the updates in regulations and accordingly changing business process models, if necessary, to support lawfulness of the system.

Figure 5 also shows an XML code fragment of one of the CC licences. It is possible to use BPMN 2.0 provided functionality to integrate XML components with the third party and to link each type of the licence used in the process (see BPMN end states in Figure 5) with its online XML based description. This will provide an opportunity for a business process modeller to access the licence using the model and developing a service for monitoring changes.

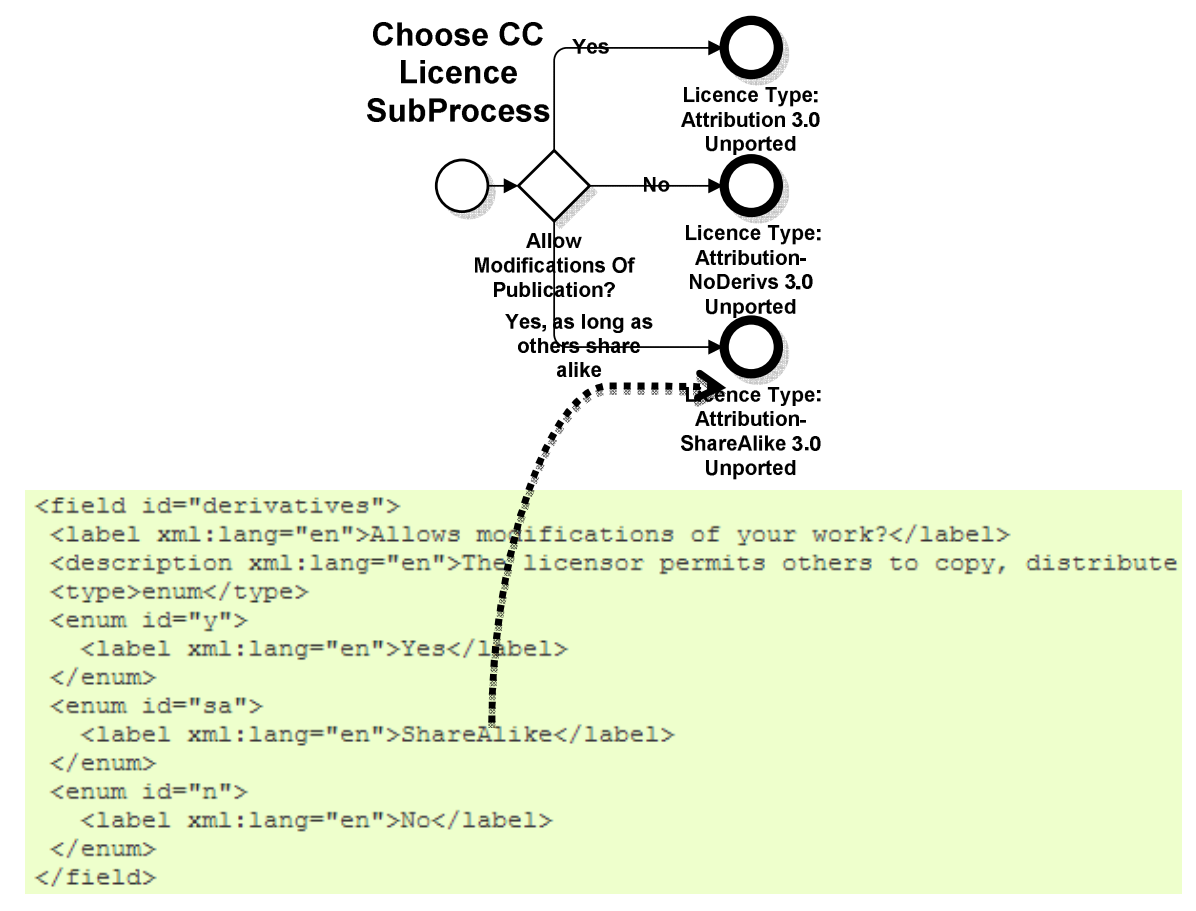

Fig. 5. "Choose CC Licence" sub-process and an XML fragment of CC licence, taken from [23]

Using the solution proposed in this paper it is possible to improve the process described in Figure 3 by automating the "Check Publisher's Policy" task that a System's Administrator must perform manually. SHERPA/ROMEO [24] is a searchable database of publisher's policies regarding the self-archiving of journal articles on the web 
and in Open Access repositories. SHERPA/ROMEO provides a machine-readable XML descriptions of publishers policies (e.g., [25]). Using the services and API provided by the SHERPA/ROMEO it is possible to automate the task of obtaining the information regarding the version of the full text the publisher permits to self-archive in institutional Open Access repository (Lawful State of the full text). After obtaining this information it is necessary to store it as a Lawful State of the Publication's full text and all other possible states of the Publication's full texts as Conceivable States. In Figure 6 we illustrate obtaining Lawful State (lawful version of publication's full text) from external XML structure (SHERPA/ROMEO XML [25]). As mentioned in this paper, BPMN and ArchiMate do not allow describing lawful aspects of the system, so using the proposed process models linkage with XML structures that allows obtaining lawful aspects of the systems and using BWW based business process knowledge repository for storing identified lawful aspects will contribute to supporting lawfulness of business process models and developing services that monitor changes in external XML documents in the real time supporting the compliance of the business process models with the newest versions of XML based regulations.

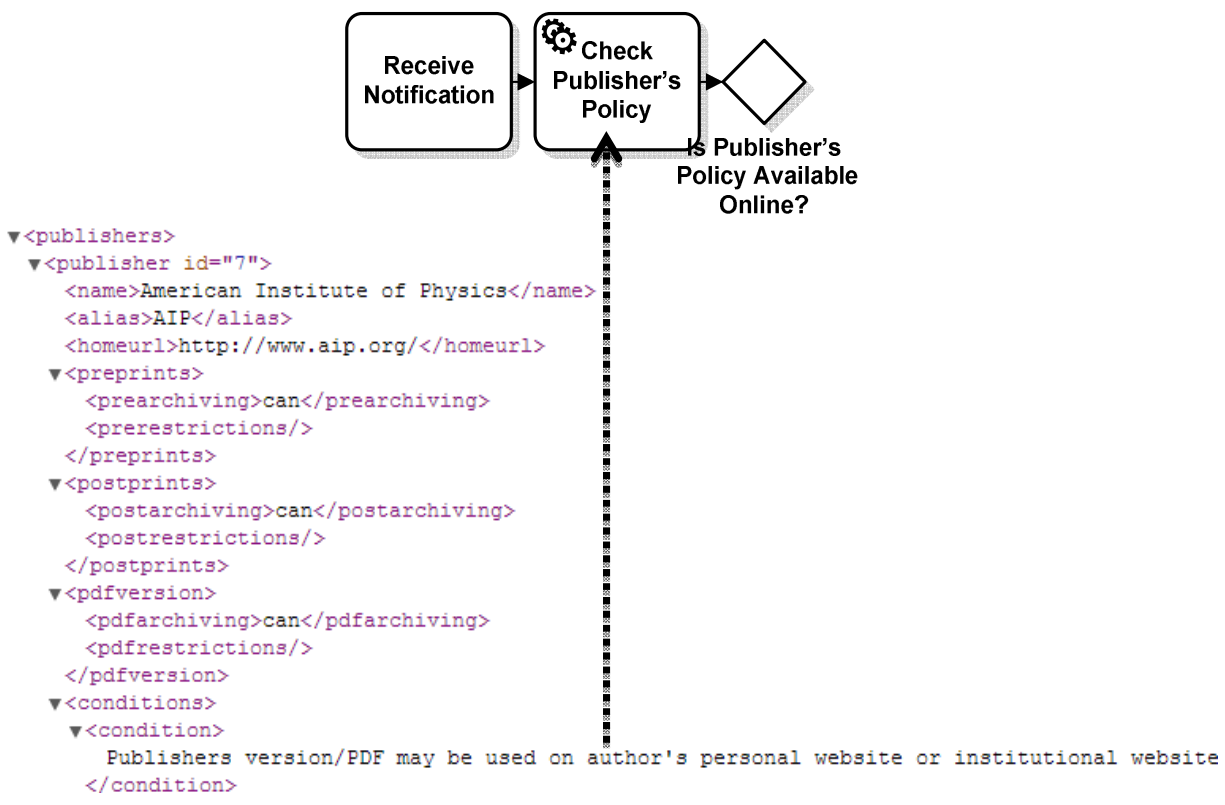

Fig. 6. Linkage between service "Check Publisher's Policy" and an XML fragment of SHERPA/ROMEO publisher's policy licence (a fragment of XML is taken from [25])

First experiments with the proposed approach show that it can contribute to the emergent challenges that organizations face in order to maintain relationships between business process, EA models, and external regulations. 


\section{Conclusions and Future Work}

This paper presented a research towards creating an efficient business process modelling environment using BWW model as the foundation for systematically storing and retrieving business process knowledge contained in interlinked BPMN models, ArchiMate models, and external XML based documents. The advantage of proposed approach resides in the following:

- BWW allows straightforwardly addressing the lawful and unlawful events and states of systems.

- BWW allows straightforwardly addressing conceivable event and state space of the system reducing the risk of including non-existing states ("unconceivable") states in the models.

BPMN and ArchiMate languages do not have elements that support identifying and storing information about lawful and unlawful states and events of the system and describing a set of conceivable states. Thus providing meaningful and accountable BPMN and ArchiMate linkage with regulations is out of the scope of these standards. BWW model provides a set of elements that can be used to store lawful and unlawful aspects of the system and link these aspects with BPMN and ArchiMate models thus providing a valuable coupling with regulations and assessing lawfulness of the system.

Therefore BPMN, ArchiMate, and XML based regulation linkage based on BWW model has capacity to close the gaps identified in this research:

1. Describing lawful and unlawful states and lawful and unlawful events of systems - neither BPMN nor ArchiMate has the ability to describe lawful and unlawful aspects of systems. Using BWW model for storing business process knowledge will provide capacity for storing lawful and unlawful aspects of the system.

2. Describing conceivable states and conceivable events of systems depicted in regulations, business process and ArchiMate models, providing possibilities to identify non-existing "unconceivable" states that might be present in the models.

BWW model based business process knowledge management contributes to creating complete and lawful business process models and systematically storing and reusing the associated business process knowledge. The presented example does not fully illustrate how the proposed approach supports the high-level concepts mentioned in the introduction (e.g., "knowledge reuse" and "holistic view of end-to-end processes"). Further research will concern more experiments with the proposed approach and development of information services to increase knowledge usage effectiveness and efficiency in business process modelling context. Also the ArchiMate 2.0 viewpoints will be analysed with regard to addressing regulations.

\section{References}

1. Grant, R.M.: Toward a Knowledge-based Theory of the Firm. Strategic Management Journal 17, 109-122 (1996)

2. Silver, B.: BPMN Method and Style with Implementer's Guide. Cody-Cassidy Press (2011) 
3. OMG: Business Process Model and Notation 2.0, http: / / www . bpmn . org

4. Businska, L., Kirikova, M., Penicina, L., Buksa, I., Rudzajs, P.: Enterprise Modeling for Respecting Regulations. PoEM Short Papers 2012. In: Emerging Topics in the Practice of Enterprise Modelling: The 5th IFIP WG8.1 Working Conference on the Practice of Enterprise Modelling (PoEM 2012), CEUR Workshop Proceedings, pp. 106-118 (2012)

5. An Official Web Site of the United States Government, http: / /www . data.gov/

6. Akoma NTOSO: XML for Parliamentary, Legislative and Juridiciary Documents, http: / / www . akomantoso.org/

7. The Open Group: ArchiMate 2.0 Specification, http://goo.gl/7gC5B

8. Wand, Y., Weber, R.: On the ontological expressiveness of information systems analysis and design grammars. Information Systems Journal 3, 217-237 (1993)

9. Recker, J., Indulska, M., Rosemann, M., Green, P.: Do Process Modelling Techniques Get Better? A Comparative Ontological Analysis of BPMN. In: Campbell, B., Underwood, J., Bunker, D. (eds.) 16th Australasian Conference on Information Systems (2005)

10. Bunge, M.: Treatise on Basic Philosophy. Ontology II: A World of Systems, vol. 4 (1979)

11. Nelson, H.J., Poels, G., Genero, M., Piattini, M.: A conceptual modeling quality framework. Software Quality Journal 20, 201-228 (2011)

12. Rosemann, M., Recker, J., Indulska, M., Green, P.: A study of the evolution of the representational capabilities of process modeling grammars. In: Martinez, F.H., Pohl, K. (eds.) CAiSE 2006. LNCS, vol. 4001, pp. 447-461. Springer, Heidelberg (2006)

13. Goumopoulos, C., Kameas, A.: Theory and Applications of Ontology: Computer Applications. Springer, Netherlands (2010)

14. zur Muehlen, M., Indulska, M., Kamp, G.: Business Process and Business Rule Modeling: A Representational Analysis. In: 2007 Eleventh International IEEE EDOC Conference Workshop, pp. 189-196. IEEE (2007)

15. Yan, Z., Dijkman, R., Grefen, P.: Business process model repositories - Framework and survey. Information and Software Technology 54, 380-395 (2012)

16. Rudzajs, P., Buksa, I.: Business Process and Regulations: Approach to Linkage and Change Management. In: Grabis, J., Kirikova, M. (eds.) BIR 2011. LNBIP, vol. 90, pp. 96-109. Springer, Heidelberg (2011)

17. Kirikova, M., Buksa, I., Penicina, L.: Raw Materials for Business Processes in Cloud. In: Bider, I., Halpin, T., Krogstie, J., Nurcan, S., Proper, E., Schmidt, R., Soffer, P., Wrycza, S. (eds.) BPMDS 2012 and EMMSAD 2012. LNBIP, vol. 113, pp. 241-254. Springer, Heidelberg (2012)

18. Softwareag: ARIS ArchiMate Modeler, http://goo.gl/WI76E

19. Chinosi, M., Trombetta, A.: BPMN: An introduction to the standard. Computer Standards \& Interfaces 34, 124-134 (2012)

20. The Open Group: TOGAF 9.1, https://www2.opengroup.org/ogsys/ catalog/g116

21. Van Den Berg, M.: ArchiMate, BPMN and UML: An approach to harmonizing the notations. Orbus software white paper (2012)

22. Creative Commons, http: / / creativecommons . org /

23. Creative Commons: CC API documentation: Version 1.0, http: / / goo.gl/I1FNC

24. SHERPA/RoMEO - Publisher copyright policies \& self-archiving, http://www . sherpa.ac.uk/romeo/

25. SHERPA/RoMEO: XML schema for Publisher's version/PDF, http://goo.gl/ OYpG6 